\title{
MicroRNA-221 inhibits CDKN1C/p57 expression in human colorectal carcinoma
}

\author{
Kai SUN*, Wei WANG, Jun-jie ZENG, Cheng-tang WU, Shang-tong LEI, Guo-xin LI \\ Department of General Surgery, Nanfang Hospital of Southern Medical University, Guangzhou 510515, China
}

\begin{abstract}
Aim: To investigate the regulatory effect of microRNA-221 (miR-221) on CDKN1C/p57 expression in colorectal carcinoma (CRC). Methods: Thirty four CRC and adjacent non-tumorous tissue samples were collected individually. Total RNA and protein were isolatedand from these samples and four human CRC-derived cell lines (including HT-29, Lovo, SW-480 and Caco2). MiR-221 expression was examined using real-time RT-PCR. CRC cells were treated with or without anti-p57-siRNA prior to the addition of premiR-221 or anti-miR-221. The mRNA and protein levels of CDKN1C/p57 were examined using semi-quantitative RT-PCR and Western blot, respectively. CRC cell proliferation and apoptosis were assessed using MTT assay and flow cytometry, respectively. The CDKN1C/p57 3'-UTR fragment was amplified using PCR from the genomic DNA of human colon cells and inserted into a luciferase reporter construct. The reporter construct was then transfected into CRC cells together with pre-miR-221 or anti-miR-221, and the luciferase activity in the transfected cells was examined.

Results: MiR-221 expression was significantly up-regulated in 90\% of CRC samples compared to that in the adjacent non-tumorous tissue, and the expression level was positively correlated to an advanced TNM stage and local invasion. There was no significant difference in CDKN1C/p57 mRNA expression between CRC and corresponding non-tumorous tissues, whereas CDKN1C/p57 protein expression was markedly decreased in the CRC samples. A significant inverse correlation between miR-221 and CDKN1C/p57 expression was found in CRC cells. Moreover, a miR-221-specific inhibitor significantly increased CDKN1C/p57 protein expression in CRC cells. Anti-miR-221 markedly inhibited CRC cell proliferation and induced apoptosis. This inhibitory effect was abolished by pretreatment with anti-p57-siRNA, suggesting that the inhibition was mediated by CDKN1C/p57. A significant increase of the luciferase activity was observed in CRC cells co-transfected with the luciferase reporter construct and anti-miR-221.

Conclusion: MiR-221 binds to the target site in the 3'-UTR of the CDKN1C/p57 mRNA to inhibit CDKN1C/p57 expression by posttranscriptional gene silencing to promote $\mathrm{CRC}$ occurrence and progress, therefore serving as a potential therapeutic target for the prevention and treatment of CRC.
\end{abstract}

Keywords: colorectal carcinoma; microRNA-221; CDKN1C/p57; proliferation; apoptosis

Acta Pharmacologica Sinica (2011) 32: 375-384; doi: 10.1038/aps.2010.206; published online 31 Jan 2011

\section{Introduction}

Colorectal carcinoma (CRC) is one of the most frequently occurring cancers and a common cause of cancer-related death worldwide, with an increasing incidence expected in the next decades ${ }^{[1]}$. The overall incidence of CRC is $5 \%$ in the general population, and the 5-year survival rate ranges from $40 \%$ to $60 \%{ }^{[2]}$. So far, prognosis largely relies on descriptive staging systems based on tumor morphology and histopathology ${ }^{[3]}$. However, morphologically similar tumors may differ in their underlying molecular changes and tumorigenic potential. CRC development from normal epithelial cells to malignant carcinomas is a multi-step process. It involves the

\footnotetext{
* To whom correspondence should be addressed.

E-mail sunkai9602@sina.com

Received 2010-08-27 Accepted 2010-11-08
}

accumulation of both genetic and epigenetic changes, leading to a temporal activation of oncogenes and an inactivation of tumor suppressor genes that confer a selective advantage to cells harboring these alterations ${ }^{[4]}$. CRC screening allows early diagnosis of the malignancy and reduces mortality. With the advent of new chemotherapeutic agents, such as angiogenesis inhibitors and TGF- $\alpha$ inhibitors, there is growing interest in identifying new prognostic biomarkers and therapeutic targets for this disease ${ }^{[5]}$.

MicroRNAs (miRNAs) are a class of small noncoding RNAs that regulate target gene expression by translational repression and mRNA cleavage/decay ${ }^{[6]}$. Genome-wide studies have demonstrated that miRNA genes are frequently located at cancer-associated genomic regions, within minimal regions of loss of heterozygosity or of amplifications, and at common breakpoint regions, indicating the potential roles of miRNAs 
in tumorigenesis ${ }^{[7]}$. MiRNAs have been demonstrated to play an important role in the multi-step process of carcinogenesis, functioning as either oncogenes or tumor suppressors. The role of miRNAs has been studied in many types of tumors, including CRC tumors. Those studies suggest that miRNAs may be potential diagnostic or prognostic tools for cancer and that identifying their target mRNAs is a key step for assessing the role of aberrantly expressed miRNAs in human cancer ${ }^{[8]}$.

MiR-221, encoded in tandem from a gene cluster located on chromosome $X$, is a recently discovered miRNA involved in tumor development by regulating cell proliferation. Fornari et $_{a l^{[9]}}$ demonstrated that miR-221 overexpression promotes hepatocellular carcinoma cell proliferation, most likely through its ability to modulate cyclin dependent kinase inhibitors (CDKIs), including CDKN1C/p57 and CDKN1B/p27. However, miR-221 expression and its targets in CRC have not been reported. CDKN1C/p57, an important regulator of cellcycle progression, belongs to the kinase inhibitor protein (CIP/ KIP) family, which has become a research focus in recent years because it can negatively regulate cell cycle progression by arresting cell proliferation in the $\mathrm{G}_{0} / \mathrm{G}_{1}$ phase ${ }^{[10]}$. CDKN1C/ p57 down-regulation in CRC has been shown to correlate with high biological aggressiveness, advanced stage, poor differentiation, large tumor size, and high proliferative activity. Furthermore, low CDKN1C/p57 expression is associated with poor prognosis and low disease-free survival after surgery, suggesting that CDKN1C/p57 down-regulation might contribute to CRC progression by regulating cell growth ${ }^{[11]}$. Under both physiological and pathological conditions, CDKN1C/p57 is mostly regulated at a post-transcriptional level ${ }^{[12]}$. Therefore, we inferred that miR-221 is up-regulated in CRC and promotes carcinogenesis by inhibiting CDKN1C/p57 expression. To test this hypothesis, we investigated the regulatory effect of miR-221 on CDKN1C/p57 expression in CRC and provided new insight into the pathogenesis, diagnosis, and treatment of CRC.

\section{Materials and methods Patients}

In total, 34 samples of CRC and matched non-tumorous tissue were harvested from patients who underwent operations in our hospital from September 2008 to May 2009 with diagnosis confirmed by postoperative pathological examination. Patients' characteristics regarding gender, age, tumor site, TNM stage, local invasion, vessel invasion, differentiation and sera carcinoembryonic antigen (CEA) were obtained from surgical and pathological records. The mean age was 52.5 years (ranging from 32 to 74 years). Local invasion was classified as tumor invading the submucosa (T1), the muscularis propria (T2), through the muscularis propria into the subserosa or nonperitonealized pericolic or perirectal tissues (T3), and through the muscularis propria into other organs or structures and/or perforated visceral peritoneum (T4). Differentiation was graded as better (including well and moderately differentiated tumors) and worse (including poorly differentiated, mucinous and signet-ring cell carcinoma). Non-tumorous tissue was harvested from the intestinal mucosa $5 \mathrm{~cm}$ from the tumor site, whereas normal controls were the intestinal mucosa over $10 \mathrm{~cm}$ from the tumor site. No patients received radiotherapy or chemotherapy before the operation. The samples were snap frozen in liquid nitrogen after collection and stored at $-80^{\circ} \mathrm{C}$. All participants of this study signed an informed consent approved by the Institutional Review Board.

\section{MiRNA target prediction}

The analysis of predicted miR-221 targets was performed using the algorithms TargetScan (http://targetscan.org/), PicTar (http://pictar.mdc-berlin.de/), and miRanda (http:// www.microrna.org/microrna/home.do) website tools.

\section{Cell culture and transfection}

Human CRC-derived cell lines, including HT-29, Lovo, SW-480 and Caco2, and control human umbilical vein endothelial cells (HUVEC), provided by Shanghai Institutes for Biological Science, were resuscitated and resuspended with RPMI-1640 supplemented with 10\% ( $v / v)$ fetal bovine serum (FBS, provided by Hyclone, USA), $100 \mathrm{U} / \mathrm{mL}$ penicillin $\mathrm{G}$ and $100 \mathrm{mg} / \mathrm{mL}$ streptomycin. The cells were then plated in $25 \mathrm{~cm}^{2}$ culture bottles and incubated in a $5 \% \mathrm{CO}_{2}$ humidified atmosphere at $37^{\circ} \mathrm{C}$. The media were changed every $3 \mathrm{~d}$, and the cells were trypsinized using trypsin-edetic acid when they reached $80 \%$ to $90 \%$ confluence. Cells aged at passages 4 to 8 were used for experiments. The day before transfection, cells were seeded in antibiotic-free medium at a density of $1 \times 10^{4}$ cells/well in 96-well plates, incubated for $24 \mathrm{~h}$, and then transfected with $80 \mathrm{nmol} / \mathrm{L}$ anti-p57 SMARTpool siRNAs or the control siRNA (Dharmacon) using Oligofectamine (Invitrogen, Carlsbad, CA, USA). Secondary transfection was performed $24 \mathrm{~h}$ after the first transfection, using $50 \mathrm{nmol} / \mathrm{L}$ negative control, anti-miR-221 negative control, pre-miR-221 or anti-miR-221, respectively (Shanghai GenePharma Co Ltd). MiRNA transfection was performed using Lipofectamine 2000 according to the manufacturer's instructions (Invitrogen). The experiment was repeated at least three times.

\section{Detection of miR-221 expression by real-time RT-PCR}

Total RNA was extracted using Trizol (Invitrogen, Carlsbad, CA, USA). The precipitation was dissolved in DEPCtreated water. A nucleic acid protein analyzer (Beckman Coulter, USA) was used to determine the RNA concentration. The purity and integrity of RNA were evaluated in formaldehyde denaturing gel electrophoresis based on two criteria: $\mathrm{A}_{260 \mathrm{~nm}} / \mathrm{A}_{280 \mathrm{~nm}} \geq 1.8$ and a band ratio of $28 \mathrm{~S}$ RNA to 18S RNA $\geq 1.5$. Mature miR-221 was accurately quantified using the TaqMan MicroRNA Assay (hsa-miR-221, N/P: 4373077; Applied Biosystems, Foster City, CA, USA). Briefly, the reverse transcription reaction was performed with $10 \mathrm{ng}$ of total RNA using the looped primers. Real-time PCR was performed on an iCycler iQ Real-Time PCR Detection System (Bio-Rad) according to the standard TaqMan MicroRNA Assay protocol. The $20-\mu \mathrm{L}$ PCR reaction included $1.33 \mu \mathrm{L}$ of reverse transcription product, $1 \times$ TaqMan Universal PCR 
Master Mix, No AmpErase UNG (P/N 4324018; Applied Biosystems), $0.2 \mu \mathrm{mol} / \mathrm{L}$ TaqMan probe, $1.5 \mu \mathrm{mol} / \mathrm{L}$ forward primer, and $0.7 \mu \mathrm{mol} / \mathrm{L}$ reverse primer. The reactions were incubated in a 96 -well plate at $95^{\circ} \mathrm{C}$ for $10 \mathrm{~min}$, followed by 40 cycles of $95^{\circ} \mathrm{C}$ for $15 \mathrm{~s}$ and $60{ }^{\circ} \mathrm{C}$ for $1 \mathrm{~min}$. MiR-221 expression was measured using $\mathrm{Ct}$ (threshold cycle). The $\mathrm{Ct}$ is the fractional cycle number at which the fluorescence of each sample passes a fixed threshold. The $\Delta \Delta \mathrm{Ct}$ method for relative quantification of gene expression was used to determine miR221 expression. The $\Delta \mathrm{Ct}$ was calculated by subtracting the $\mathrm{Ct}$ of RNU6B from that of miR-221. The $\Delta \Delta \mathrm{Ct}$ was calculated by subtracting the $\Delta \mathrm{Ct}$ of the reference sample from that of each tested sample. Fold change was calculated using the equation of $2^{-\Delta \Delta \mathrm{Ct}}$. The TaqMan MicroRNA Assay for U6 RNA (RNU6B, P/N: 4373381; Applied Biosystems) was used to normalize the relative abundance of miR-221. The experiment was repeated at least three times.

\section{Detection of CDKN1C/p57 mRNA expression by semi-quanti- tative RT-PCR}

Briefly, $3 \mu \mathrm{g}$ of total RNA was used as the template for RTPCR analysis according to the instructions of the RNA PCR Kit Ver 3.0 of TaKaRa Company. The PCR parameters were as follows: $94{ }^{\circ} \mathrm{C}$ for $2 \mathrm{~min} ; 30$ cycles of $94^{\circ} \mathrm{C}$ for $30 \mathrm{~s}, 55^{\circ} \mathrm{C}$ for $30 \mathrm{~s}$, and $72{ }^{\circ} \mathrm{C}$ for $1 \mathrm{~min}$; and $72{ }^{\circ} \mathrm{C}$ for $10 \mathrm{~min}$. Of the PCR products, $10 \mu \mathrm{L}$ was loaded on a $1.5 \%$ agarose gel stained with ethidium bromide $(0.5 \mathrm{~g} / \mathrm{L})$. The signal was quantitated by densitometry using the Image Master VDS system and the associated software (Pharmacia, USA) as follows: relative mRNA content=integral absorbance value of the target gene band/integral absorbance value of the internal reference GAPDH. The experiment was repeated at least three times ${ }^{[13]}$.

\section{Detection of CDKN1C/p57 protein expression by Western blot}

The tissues and cells were rinsed twice with cold PBS buffer and lysed in an ice-cold lysis buffer containing $150 \mathrm{mmol} / \mathrm{L}$ $\mathrm{NaCl}, 50 \mathrm{mmol} / \mathrm{L}$ Tris-HCl (pH 7.6), 0.1\% SDS, 1\% Nonidet $\mathrm{P}-40$, and a protease inhibitor cocktail (Boehringer Mannheim, Lewes, UK). The samples were cleared by centrifugation at $13000 \times \mathrm{g}$ for $10 \mathrm{~min} ; 50 \mu \mathrm{g}$ of protein from the tissue samples was subjected to sodium dodecyl sulfate-polyacrylamide gel electrophoresis (SDS-PAGE) and electrotransferred to polyvinylidene fluoride (PVDF) membranes (Immobilon, Bedford, MA). After being blocked in $20 \mathrm{mmol} / \mathrm{L}$ Tris- $\mathrm{HCl}, \mathrm{pH}$ 7.6 (containing $150 \mathrm{mmol} / \mathrm{L} \mathrm{NaCl}, 0.1 \%$ Tween-20, and $5 \%$ non-fat dry milk), the membranes were incubated with primary antibodies against CDKN1C/p57 or $\beta$-actin (used as a loading control) overnight at $4{ }^{\circ} \mathrm{C}$ and then incubated with a horseradish peroxidase-conjugated secondary antibody. The blot was developed using an ECL detection kit (Amersham Pharmacia Biotech), according to the manufacturer's instructions, and the protein imprinting band was obtained. The experiment was repeated at least three times.

Cell proliferation analysis of transfected cells by the MTT assay Cell proliferation was determined by the 3-(4,5-dimethylthi- azol-2-yl)-2,5-diphenyl tetrazolium bromide (MTT provided by Amresco, USA) assay. Briefly, exponentially growing CRC cells were adjusted to $1.5 \times 10^{4}$ cells $/ \mathrm{mL}$ with RPMI1640 , plated in 96-well plates (Corning, USA) at $200 \mu \mathrm{L} /$ well and then incubated for $12 \mathrm{~h}$. After being transfected with 50 $\mathrm{nmol} / \mathrm{L}$ miR-221 or anti-miR-221 and incubated for $48 \mathrm{~h}$ (five duplicate wells for each sample), $20 \mu \mathrm{L} /$ well MTT ( $5 \mathrm{~g} / \mathrm{L}$ ) was added to each well. The medium was then removed after a 4-h incubation, and $100 \mu \mathrm{L} /$ well dimethyl sulfoxide (DMSO) was added to dissolve the reduced formazan product. Subsequently, the plate was read in an enzyme-linked immunity implement (BIO-RAD2550, USA) at $490 \mathrm{~nm}$. Cellular proliferation inhibition rate (CPIR) was calculated according to the following formula: $\mathrm{CPIR}=(1$-average $\mathrm{A}$ value of the experimental group/average A value of the control group) $\times 100 \%$. The experiment was repeated at least three times.

\section{Cell-cycle and apoptosis analyses of transfected cells by flow} cytometry

The effects of miR-221 on cell cycle and apoptosis in CRC cells were examined by flow cytometry. In brief, pretreated CRC cells were harvested and washed twice with PBS buffer, fixed with $70 \%$ ethanol at $-20^{\circ} \mathrm{C}$ for $30 \mathrm{~min}$ and stored at $4^{\circ} \mathrm{C}$ overnight. The cells were then washed with PBS again, treated with $100 \mathrm{~mL}$ of $100 \mathrm{mg} / \mathrm{L}$ RNase at $37^{\circ} \mathrm{C}$ for $30 \mathrm{~min}$ and stained with Annexin V-FITC and PI at $4{ }^{\circ} \mathrm{C}$ for $30 \mathrm{~min}$ in darkness. Cell cycle and apoptotic rate were measured using an EPICS XL Flow Cytometer (Coulter, USA) at $488 \mathrm{~nm}$, and the data were analyzed using the CELL Quest Software. The percentages of $G_{0} / G_{1}$ phase, $S$ phase and the apoptotic rate were measured by calculating the ratio of the number of corresponding cells and that of total cells. For each sample, 10000 cells were measured. The experiment was repeated at least three times.

\section{Luciferase activity assay}

The human 3'-untranslated region (3'-UTR) of the CDKN1C/ p57 gene was amplified by PCR and cloned into the XbaI site of the pGL3-Control vector (Promega, Madison, WI, USA), downstream of the luciferase gene, to generate the vector pGL3-p57. For the luciferase assay, the CRC cells were cultured in 24-well plates and were transfected with $500 \mathrm{ng}$ of either pGL3-p57 or the pGL3 control vector and 50 pmol of miR-221, anti-miR-221 or negative controls. Transfection was performed using Lipofectamine 2000 (Invitrogen) according to the manufacturer's instructions. At $24 \mathrm{~h}$ after transfection, the firefly luciferase activity was measured using the DualLuciferase Reporter Assay (Promega). The experiment was repeated at least three times.

\section{Statistical analysis}

The relative expression of target genes was analyzed using REST-XL (Relative Expression Software Tool, available at http://www.gene-quantification.de/rest.html). All data were presented as the average \pm standard deviation (Mean \pm SD). Comparisons between groups were performed by one-way 
ANOVA and Student-Newman-Keuls $q$ test using SPSS 13.0 (SPSS Inc, Chicago, IL, USA). $P$ values less than 0.05 were considered statistically significant.

\section{Results}

CDKN1C/p57 mRNA expression is down-regulated in some CRC samples

To investigate the regulatory effect of miR-221 on CDKN1C/ p57 protein expression in CRC, we examined CDKN1C/ p57 mRNA expression in 34 CRC samples by semi-quantitative RT-PCR. The data showed that in four CRC samples, CDKN1C/p57 mRNA expression significantly decreased compared with the adjacent non-tumorous tissue (Figure 1A). These cases were excluded from the following experiments, whereas the remaining thirty samples (whose CDKN1C/p57 mRNA expression showed no significant difference between $\mathrm{CRC}$ and the adjacent non-tumorous tissue) were used for the following study (selective cases were shown in Figure 1B).

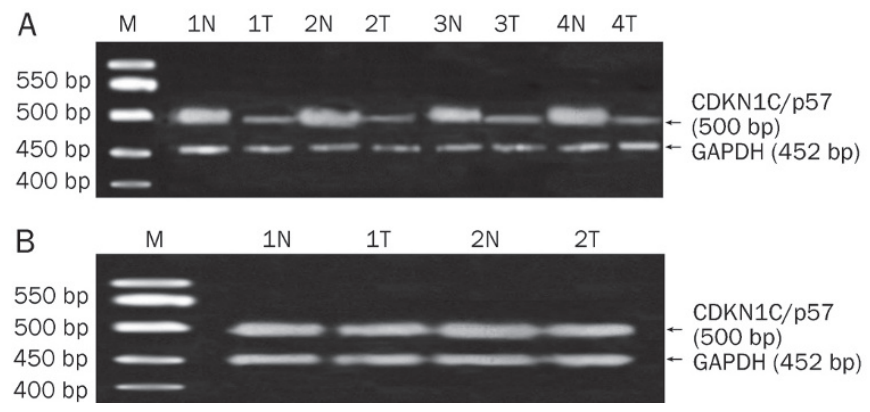

Figure 1. Semi-quantitative RT-PCR analysis showing CDKN1C/p57 mRNA expression in several CRCs and adjacent non-tumorous tissues. A reduction of CDKN1C/p57 mRNA levels can be observed in four CRC samples compared with adjacent non-tumorous tissues (A). The remaining thirty samples showed no significant difference of CDKN1C/ p57 mRNA expression in CRC and adjacent non-tumorous tissues (selective cases were reported in (B). Equal loading was confirmed by using GAPDH as a loading control. M: Marker; N, T represented the patients' nontumorous tissues $(\mathrm{N})$ and $\mathrm{CRCs}(\mathrm{T})$, respectively.

MiR-221 is aberrantly overexpressed in CRC tissues and CRCderived cell lines

To study the expression pattern of miR-221 in CRC, we used real-time RT-PCR to examine miR-221 expression in thirty CRC samples and the adjacent non-tumorous tissue, as well as in four CRC-derived cell lines. The data showed that the miR221 cDNA level exponentially increased and reached the plateau phase. Its amplification curve displayed a typical reverse $S$ pattern (Figure 2A), indicating high amplification efficiency. The miR-221 PCR product was $73 \mathrm{bp}$ in length, and the corresponding Tm was $84.26 \pm 0.56^{\circ} \mathrm{C}$. The melting temperature was even, and the shape of the peak was sharp (Figure 2B). Of the 30 cases, 27 (90\%) showed significantly up-regulated miR221 expression in CRC compared with that in the non-tumorous tissue $(2.041 \pm 1.401$ vs $0.706 \pm 0.341, P<0.01)$. As shown
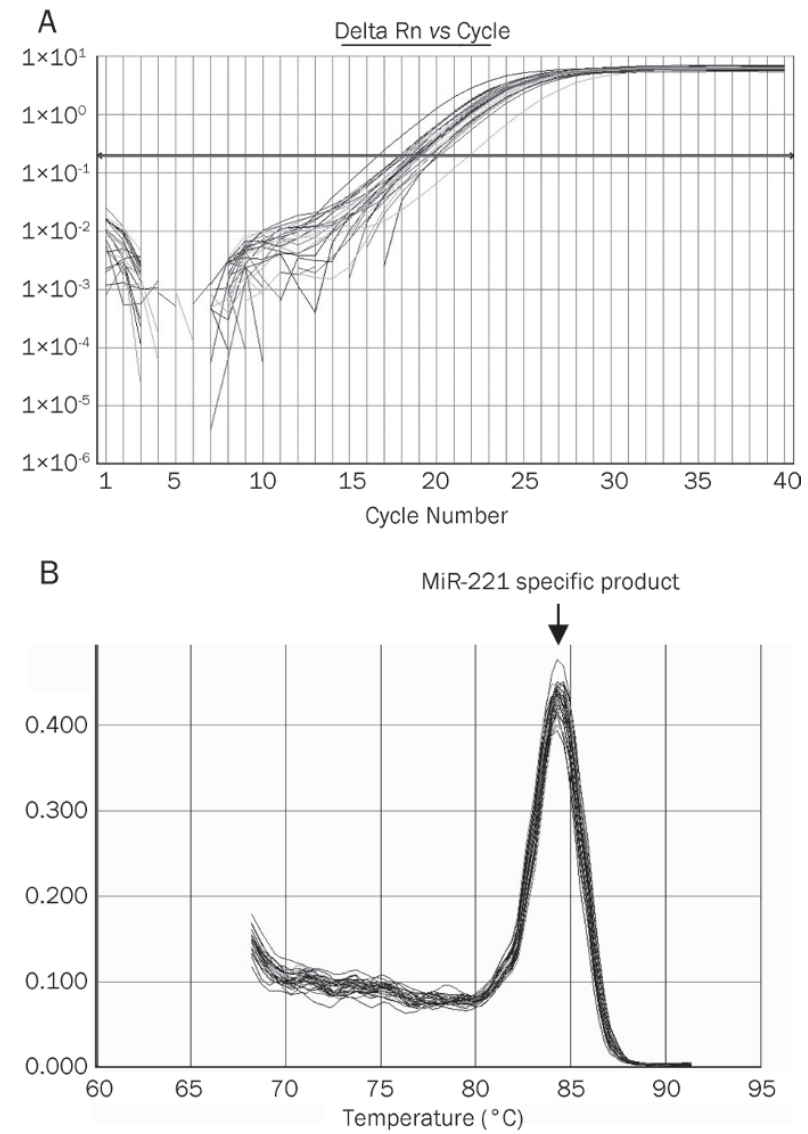

Figure 2. Real-time RT-PCR analysis detecting miR-221 expression in CRCs and adjacent non-tumorous tissues. (A) miR-221 cDNA concentrations' Log value as ordinate, Ct value as abscissa. (B) Tm of miR-221 was $84.26^{\circ} \mathrm{C}$.

in Table 1, miR-221 expression was related to TNM stage $(P=0.032)$ and local invasion $(P=0.021)$. MiR-221 expression was higher in tumors at a more advanced TNM stage and with deeper invasions. The expression levels of miR-221 in HT-29, Lovo, SW-480, and Caco2 were 4.094 $\pm 0.208,1.122 \pm 0.138$, $3.927 \pm 0.232$, and $1.831 \pm 0.149$, respectively. Significant miR-221 overexpression was observed in all four CRC cell lines compared to the miR-221 level in HUVEC $(0.223 \pm 0.047, P<0.01)$. The Caco2 cell line was chosen for both miR-221 and antimiR-221 transfection in the subsequent experiment because of its intermediate miR-221 expression level among the four tested cell lines.

\section{CDKN1C/p57 is a target of miR-221 in CRC}

To confirm the regulatory effect of miR-221 on CDKN1C/ p57 in CRC, we selected a group of samples with similar CDKN1C/p57 mRNA expressions between CRC and the matched non-tumorous tissue to avoid the $20 \%-25 \%$ of CRC cases in which CDKN1C/p57 is affected by aberrant methylation at chromosome 11p15.5. Among the 34 initial samples of CRC and matched non-tumorous tissue, 30 cases were selected because they displayed similar CDKN1C/p57 mRNA levels. 
Table 1. Relationship between miR-221 expression and clinicopathological features in CRC patients.

\begin{tabular}{|c|c|c|c|}
\hline Variable & NO & miR-221 (Mean $\pm S D)$ & $P$ value \\
\hline Gender & & & 0.096 \\
\hline Male & 16 & $2.154 \pm 1.620$ & \\
\hline Female & 14 & $1.800 \pm 0.714$ & \\
\hline Age & & & 0.605 \\
\hline$\leq 50$ & 11 & $1.898 \pm 0.976$ & \\
\hline$>50$ & 19 & $2.145 \pm 1.457$ & \\
\hline Site & & & 0.586 \\
\hline Colon & 12 & $1.935 \pm 0.577$ & \\
\hline Rectum & 18 & $2.037 \pm 1.033$ & \\
\hline TNM stage & & & 0.032 \\
\hline I+II & 11 & $1.185 \pm 0.392$ & \\
\hline III+IV & 19 & $2.658 \pm 1.437$ & \\
\hline Local invasion & & & 0.021 \\
\hline $\mathrm{T} 1+\mathrm{T} 2+\mathrm{T} 3$ & 23 & $1.275 \pm 0.445$ & \\
\hline $\mathrm{T} 4$ & 7 & $2.833 \pm 1.498$ & \\
\hline Differentiation & & & 0.767 \\
\hline Better & 16 & $2.168 \pm 1.128$ & \\
\hline Worse & 14 & $1.974 \pm 0.824$ & \\
\hline CEA ( $\mu \mathrm{g} / \mathrm{L})$ & & & 0.429 \\
\hline$\leq 5$ & 10 & $2.020 \pm 0.321$ & \\
\hline$>5$ & 20 & $2.213 \pm 0.647$ & \\
\hline
\end{tabular}

To assess the correlation between miR-221 expression and the CDKN1C/p57 protein level in CRC, we examined miR221 expression by real-time RT-PCR and analyzed CDKN1C/ p57 protein expression by Western blot in the 30 matched samples of CRC and adjacent non-tumorous tissue, among which $27(90 \%)$ showed down-regulation of CDKN1C/p57 protein expression in CRC compared to that in the matched non-tumorous tissue $(3.019 \pm 1.708$ vs $0.972 \pm 0.31, P<0.01)$. Interestingly, the down-regulation was not observed at the mRNA level, indicating that the protein was down-regulated at a post-transcriptional level (selective cases were shown in Figure 3A). As shown in Figure 3B, when CDKN1C/p57 protein expression was plotted against miR-221 expression, an inverse correlation was observed $\left(r^{2}=0.904, P<0.01\right)$. Together, these results suggest that miR-221 may negatively regulate CDKN1C/p57 in CRC cells.

We further examined the effect of miR-221 on CDKN1C/ p57 expression in CRC cells by ectopic expression of miR-221. Briefly, we transfected pre-miR-221 or its inhibitor, a specific 2'-methoxy-modified RNA oligonucleotide (anti-miR-221), into Caco2 cells, and we examined CDKN1C/p57 protein expression by Western blot. Ectopic expression of miR-221 caused a significant increase of miR-221 expression $(2.915 \pm 0.442)$ and a decrease of CDKN1C/p57 protein expression. Conversely,
A
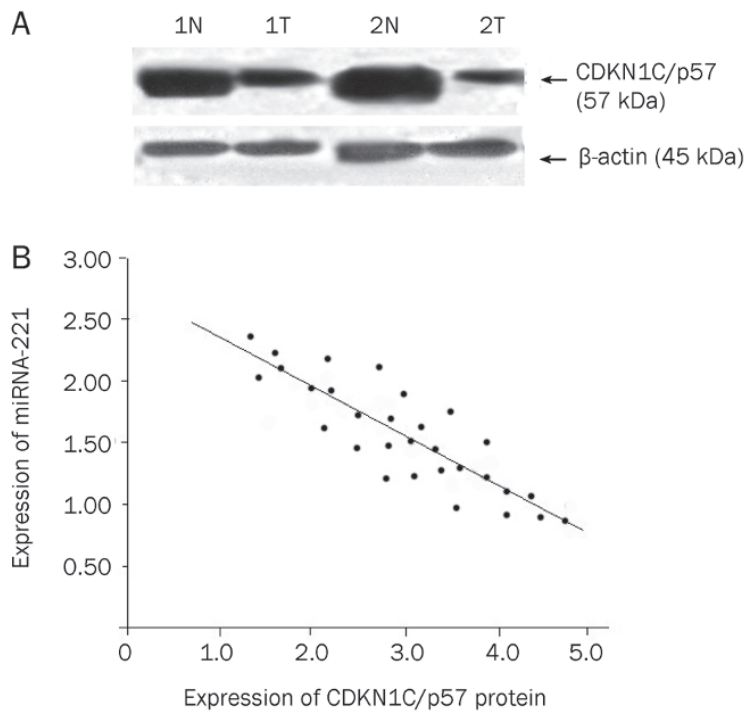

Figure 3. Inverse correlation between miR-221 and CDKN1C/p57 in $\mathrm{CRC}$ samples. (A) CDKN1C/p57 protein levels in CRC and matched nontumorous tissues. A reduction of $\mathrm{CDKN} 1 \mathrm{C} / \mathrm{p} 57$ protein levels can be observed in several CRCs (total proteins, from the same samples utilized for RT-PCR in Figure 1B, were analyzed by Western-blot with anti-p57 antibodies. $\beta$-actin was used as house-keeping gene to normalize CDKN1C/p57 protein expression). Note: N, T represented the patients' non-tumorous tissues $(\mathrm{N})$ and $C R C s(T)$, respectively. (B) Inverse correlation between miR-221 and CDKN1C/p57 in CRC tissues. MiR-221 quantitation was obtained by real-time RT-PCR, whereas CDKN1C/p57 protein expression was evaluated by Western-blot analysis. A statistically significant inverse correlation between miR-221 and CDKN1C/p57 protein levels was observed $(P<0.01)$.

anti-miR-221 induced a significant decrease of miR-221 expression $(0.541 \pm 0.206)$ and an increase of CDKN1C/p57 protein expression (Figure $4 \mathrm{~A}$ ). Interestingly, no significant changes in CDKN1C/p57 mRNA expression were observed in the

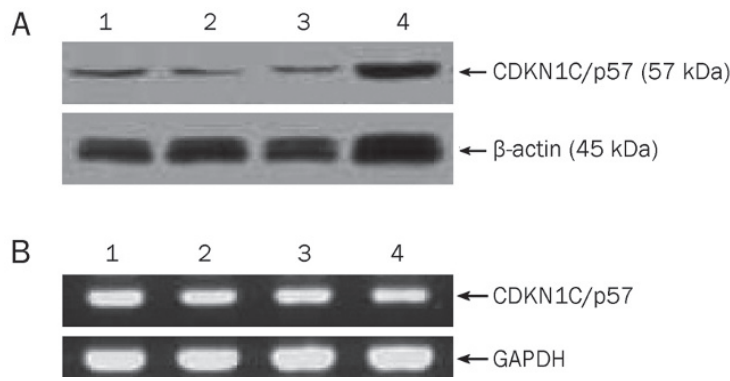

Figure 4. MiR-221 regulates the expression of CDKN1C/p57 in CRCderived cells. (A) Western-blot analysis showing CDKN1C/p57 protein expression in Caco2 cells. $\beta$-actin was used as house-keeping gene to normalize CDKN1C/p57 protein expression. (B) Semi-quantitative RT$\mathrm{PCR}$ analysis showing CDKN1C/p57 mRNA expression in Caco2 cells. The transfected cells showed no significant difference of CDKN1C/p57 mRNA expression. Equal loading was confirmed by using GAPDH. Note: lane 1, negative control; lane 2, anti-miR-221 negative control; lane 3, miR-221; lane 4, anti-miR-221. The results are representative of three independent experiments. 
cells transfected with either miR-221 or anti-miR-221 (Figure $4 \mathrm{~B})$. These results strongly indicate that miR-221 regulates CDKN1C/p57 protein expression at a post-transcriptional level but does not cause CDKN1C/p57 mRNA degradation.

\section{MiR-221 interacts with the $3^{\prime}-\mathrm{UTR}$ of the CDKN1C/p57 mRNA}

Most miRNAs are thought to regulate gene expression by base-pairing with the miR-recognizing elements (miR-RE) in their target mRNAs. Therefore, we used all of the three currently available major prediction programs, including TargetScan, Miranda and PicTar, to analyze the potential interaction between miR-221 and CDKN1C/p57. The CDKN1C/p57 mRNA was predicted as a miR-221 target by at least two of the algorithms, and a potential miR-221 target site was predicted in its $3^{\prime}$-UTR region (Figure 5A). To confirm that the direct interaction between miR-221 and the CDKN1C/p57 mRNA was responsible for the decreased CDKN1C/p57 expression, we cloned a 262-bp CDKN1C/p57 3'-UTR fragment, including the potential target site for miR-221, downstream of the pGL3 luciferase reporter gene to generate the pGL3-p57 vector. This vector was co-transfected with miR-221 or anti-miR-221 into Caco2 cells. The luciferase activity in Caco2 cells cotransfected with the pGL3-p57 vector and miR-221 markedly decreased compared with that in the negative control. On the contrary, the luciferase activity in $\mathrm{Caco} 2$ cells transfected with anti-miR-221 significantly increased compared with that in the negative control (Figure 5B). These results support the bioinformatic prediction indicating the $3^{\prime}$-UTR of CDKN1C/p57 mRNA as a miR-221 target.

\section{MiR-221 increases cell proliferation and inhibits apoptosis in CRC cells}

Because CDKN1C/p57 plays a key role in cell cycle progression, particularly in cell growth arrest at the $G_{1} / S$ transition, we further tested if the growth of stably transfected CRC cells expressing miR-221was affected as a result of the demonstrated CDKN1C/p57 reduction. First, to evaluate the effect of miR-221 on CRC cell proliferation, we transfected Caco2 cells with miR-221 or anti-miR-221. At $48 \mathrm{~h}$ after transfec- tion, cell proliferation was determined by the MTT assay. We observed a significant increase in the proliferation rate after the transfection of miR-221. In contrast, anti-miR-221 significantly decreased cell proliferation (Figure 6A, $P<0.01$ ). These data indicate that increased miR-221 expression significantly promotes cell proliferation in Caco2 cells. Second, we investigated the cell cycle phase distribution in CRC after the transfection of miR-221 or anti-miR-221 by flow cytometry. The results showed a significant increase in the S-phase population and a decrease in the $G_{0} / G_{1}$ population in Caco2 cells overexpressing miR-221 compared with the cells transfected with the negative control. On the contrary, anti-miR-221 significantly decreased the S-phase population and increased the $\mathrm{G}_{0} / \mathrm{G}_{1}$ population (Figure $6 \mathrm{~B}, P<0.01$ ). Third, we investigated the effect of anti-miR-221 on cell apoptosis by flow cytometry and found that cell apoptosis dramatically increased in Caco2 cells after transfection with anti-miR-221, suggesting that antimiR-221 may function as a strong apoptosis inducer in human CRC cells (Figures 6C-6D). These results strongly support the potential oncogenic activity of miR-221 in CRC.

\section{CRC cell proliferation suppression by anti-miR-221 is mediat- ed by CDKN1C/p57}

If the suppression of CRC cell proliferation by anti-miR-221 is indeed mediated by CDKN1C/p57, we would expect the suppression effect to be abolished by the CDKN1C/p57specific irreversible antagonist anti-p57-siRNA. To test this hypothesis, we measured the effect of miR-221 and antimiR-221 on cell proliferation in CRC cells previously transfected with anti-p57-siRNA. The aim of this experiment was to address if and how the CDKN1C/p57-depleted cellular environment responds to miR-221 or anti-miR-221 overexpression. Briefly, Caco2 cells were pretreated with or without anti-p57-siRNA $(80 \mathrm{nmol} / \mathrm{L})$ for $24 \mathrm{~h}$ prior to the addition of pre-miR-221 (50 nmol/L) or anti-miR-221 (50 nmol/L). Cell proliferation was determined by MTT assay. The data showed that CDKN1C/p57 down-regulation by means other than miR-221 overexpression led to an analogous outcome: when Caco2 cells were transfected with anti-p57-siRNA, caus-

\begin{tabular}{|c|c|c|c|}
\hline $\begin{array}{l}\text { Target } \\
\text { gene }\end{array}$ & TargetScan prediction & $\begin{array}{l}\text { Predicted consequential pairing of } \\
\text { target region (top) and miRNA (bottom) }\end{array}$ & $\begin{array}{l}\text { Seed } \\
\text { match }\end{array}$ \\
\hline CDKN1C & $\begin{array}{l}\text { Position } 86-92 \text { of CDKN1C } \\
\text { 3'-UTR hsa-miR-221 }\end{array}$ & $\begin{array}{l}5^{\prime} \quad \ldots \text { CGGCUGGGACCGUUCAUGUAGCA ... } \\
3^{\prime} \text { CUUUGGGUCGUCUGU - UACAUCGA }\end{array}$ & 8 mer \\
\hline
\end{tabular}

B

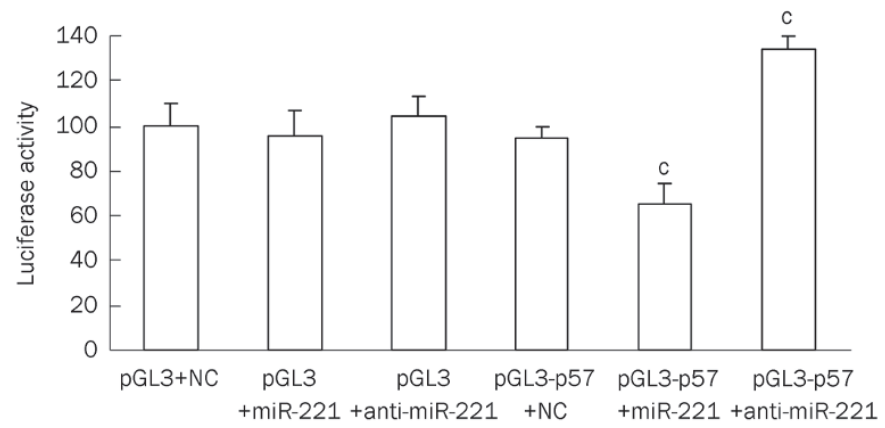

Figure 5. Direct regulation of CDKN1C/p57 mRNA by miR221. (A) CDKN1C/p57 3'-UTR site potentially targeted by miR-221 as predicted by TargetScan. (B) Luciferase activity assay showing direct interaction between miR-221 and CDKN1C/p57 3'-UTR site. Firefly luciferase reporter activity on the presence of both pGL3-p57 vector and miR221 or anti-miR-221 were compared with those of the controls. Luciferase activity in Caco2 cells co-transfected with pGL3-p57 vector and miR-221 was decreased markedly compared with negative control. On the contrary, luciferase activity in Caco 2 cells transfected with anti-miR-221 was increased significantly compared with negative control. $n=3$. Mean \pm SD. ${ }^{c} P<0.01$ vs control group. 
A

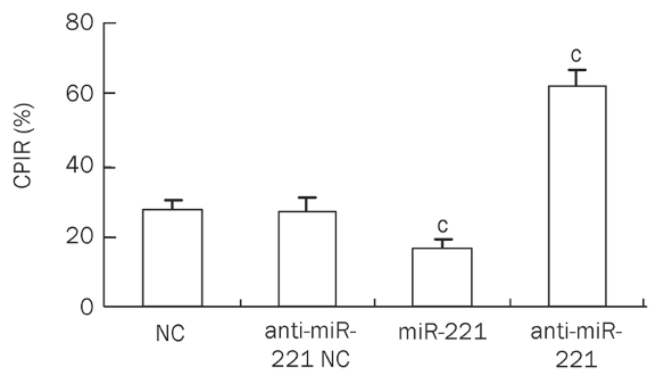

C

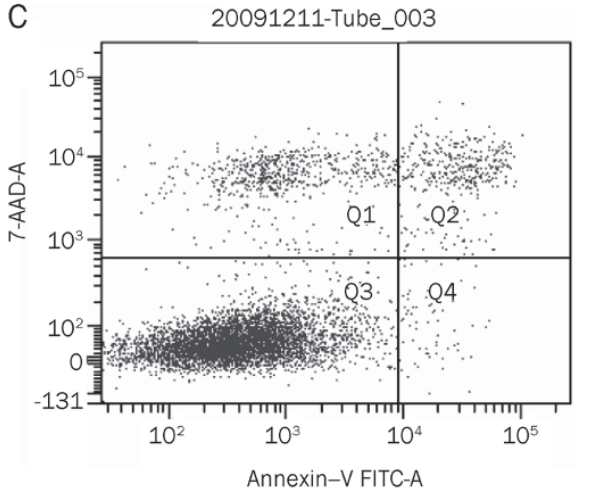

B

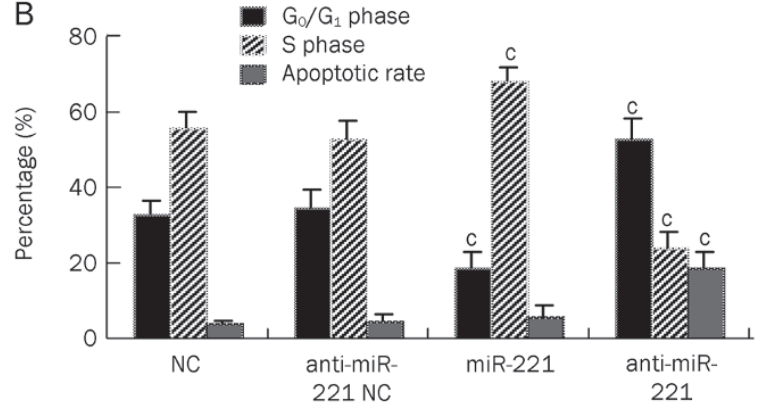

D

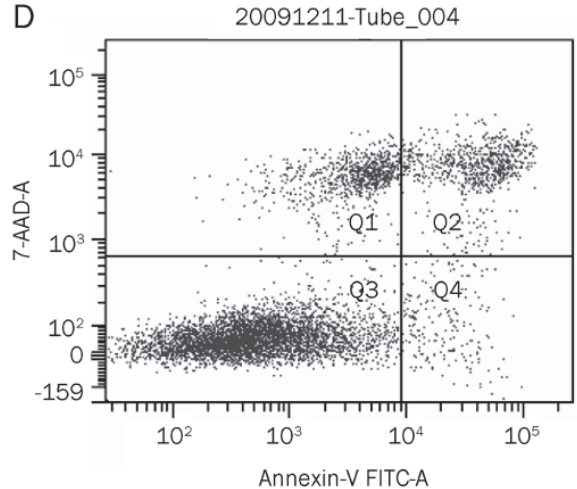

Figure 6. Effects of miR-221 and anti-miR-221 on cell proliferation and apoptosis in CRC cells. (A) The status of cell proliferation was determined by MTT assay. CPIR on the presence of miR-221 or anti-miR-221 was compared with those of the controls. $n=6$. Mean \pm SD. ${ }^{c} P<0.01$ vs control group. (B) Effects of miR-221 and anti-miR-221 on cell-cycle in Caco2 cells. The percentages of $G_{0} / G_{1}$ phase, $S$ phase and apoptotic rate were measured by computing the ratio of the number of corresponding cells and that of total cells. $n=3$. Mean \pm SD. ${ }^{c} P<0.01$ vs control group. (C, D) Cell apoptosis analysis of transfected cells by flow cytometer. Note: C, NC group; D, anti-miR-221 group. The right-down quadrant (FITC $\left.{ }^{+} / \mathrm{PI}^{-}\right)$was shown as apoptotic cells.

ing about $70 \%$ down-regulation of CDKN1C/p57 at both the mRNA and the protein levels (Figure 7A-7B), we observed a marked increase in cell proliferation compared with the negative control (Figure 7C). Thus, reducing CDKN1C/p57 protein expression in CRC cells, by either miR-221 overexpression or anti-p57-siRNA transfection, promoted cell proliferation at a comparable level.

When miR-221 was transfected into Caco2 cells previously treated with anti-p57-siRNA, we observed that, at $48 \mathrm{~h}$ after miR-221 transfection, anti-p57-siRNA and miR-221 seemed to cooperate to increase the growth rate (Figure 7C). Conversely, when anti-miR-221 was transfected into Caco2 cells previously treated with anti-p57-siRNA, we observed that, at $48 \mathrm{~h}$ after anti-miR-221 transfection, the suppression of cell proliferation by anti-miR-221 was largely abrogated by anti-p57siRNA (Figure 7C). These results indicate that the inhibitory effect of anti-miR-221 on CRC cell growth is largely, but not completely, mediated by CDKN1C/p57, suggesting that anti-miR-221 may also activate CDKN1C/p57-independent signaling pathways to inhibit CRC cell growth.

\section{Discussion}

In this study, we identified CDKN1C/p57 as a direct target of miR-221 in CRC. The data have shown that in CRC, miR-
221 is up-regulated and CDKN1C/p57 protein expression is down-regulated. A significant inverse correlation between miR-221 and CDKN1C/p57 was observed in CRC. CDKN1C/ p57 modulation by miR-221 was demonstrated by in vitro transfection experiments in CRC-derived cell lines and by confirming the direct interaction between miR-221 and the CDKN1C/ p57 3'-UTR target site using the luciferase assay.

MiR-221 is a newly discovered miRNA that is up-regulated in multiple malignancies such as hepatocellular carcinoma, bladder cancer, and pancreatic cancer ${ }^{[14-16]}$. It promotes tumor cells entering the $S$ phase from the $G_{0} / G_{1}$ phase by inhibiting CDKI expression ${ }^{[17,18]}$. Therefore, miR-221 is an attractive candidate for selective treatment using miR-221-specific inhibitors. A few published reports have begun to reveal the mechanisms that may link miR-221 up-regulation to cancer; however, the exact underlying molecular mechanisms remain unclear. CDKN1C/p57 is a negative regulatory factor of cell cycle dependent kinases that belong to the CIP/KIP family. It was first cloned using a two-hybrid system by Matsuoka et $a l^{[19]}$ in 1995 and was located within the region of about 22 $\mathrm{kb}$ in length between D11S648 and D11S679 on chromosome 11p15.5. The a 2 helix domain at the amino terminus of its product, the $\mathrm{p} 57^{\mathrm{kip} 2}$ protein, can form homodimers, which in turn interact with the cyclin2-CDK4 complex and inhibit 
A

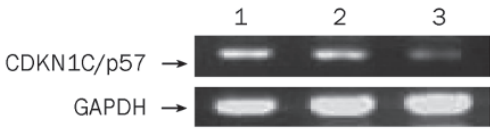

B

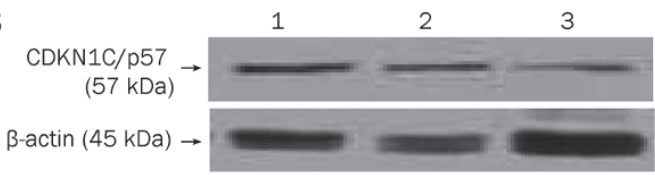

C

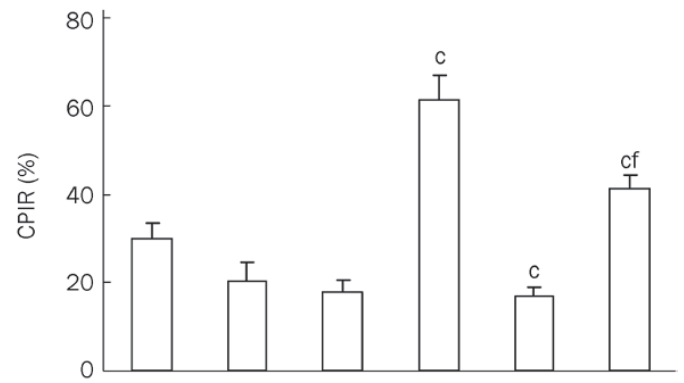

Anti p57-siRNA miR-221 Anti-miR-221

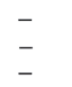

$+$

$-$

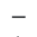

-
+
-

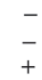

$\begin{array}{ll}+ & + \\ + & -\end{array}$

Figure 7. Ectopic expression of miR-221 affects the growth of CRC cells by targeting $\mathrm{CDKN} 1 \mathrm{C} / \mathrm{p} 57$. Caco2 cells were pretreated with or without anti-p57-siRNA ( $80 \mathrm{nmol} / \mathrm{L})$ for $24 \mathrm{~h}$ prior to the addition of pre-miR-221 $(50 \mathrm{nmol} / \mathrm{L})$ or anti-miR-221 $(50 \mathrm{nmol} / \mathrm{L})$. Semi-quantitative RT-PCR and Western-blot analysis showing that both CDKN1C/p57 mRNA (A) and protein $(B)$ reduced markedly after transfection with anti-p57-siRNA. Equal loading was confirmed by using GAPDH or $\beta$-actin. Note: lane 1 , negative control; lane 2, Caco2 cells transfected with control siRNA; lane 3, Caco2 cells transfected with anti-p57-siRNA. (C) The status of cell proliferation was determined by MTT assay. The suppression of Caco2 cell proliferation by anti-miR-221 was largely, but not completely, abrogated by anti-p57-siRNA (CPIR from $61.4 \%$ to $41.2 \%$ ). $n=3$. Mean \pm SD. ${ }^{c} P<0.01$ vs negative control group. ${ }^{f} P<0.01$ vs sole anti-miR-221 group.

the complex function to prevent cell cycle progression from the $G_{0} / G_{1}$ phase to the $S$ phase. Indeed, reduced or absent CDKN1C/p57 expression has been shown in many types of human cancers to correlate with tumor aggressiveness, poor prognosis and high recurrence rate. Soejima et al ${ }^{[20]}$ have demonstrated that the loss of imprinting (LOI) of the methylation of the maternal allele at the KvDMR1 locus occurs in about $20 \%$ to $25 \%$ of malignant cells and that the KvDMR1 locus is also at chromosome $11 \mathrm{p} 15.5$, which is the locus of the CDKN1C/p57 gene that causes CDKN1C/p57 down-regulation. In the present study, we found that $\mathrm{CDKN} 1 \mathrm{C} / \mathrm{p} 57$ protein expression is down-regulated in about $90 \%$ of unscreened CRC samples in our preliminary experiment, suggesting that CDKN1C/p57 down-regulation might have additional outcomes other than aberrant methylation. Moreover, the regulation of CDKN1C/p57 expression and function essentially occurs at the post-transcriptional level ${ }^{[21]}$. With the application of bioinformatic predictions using TargetScan, PicTar and miRanda, we found that miR-221 and the CDKN1C/p57
mRNA 3'-UTR have complementary binding sites, suggesting that CDKN1C/p57 might be a novel target of miR-221 in CRC that has not yet been reported.

In this study, we showed that CDKN1C/p57 mRNA expression was low in about $11.8 \%(4 / 34)$ of CRC cases, in agreement with the previous hypothesis of loss of imprinting of the maternal gene allele methylation in tumor cells and the downregulation of CDKN1C/p57 mRNA expression at the transcriptional level. However, the subsequent down-regulation of CDKN1C/p57 protein expression is probably not caused by the post-transcriptional gene silencing of miR-221. These samples were excluded from the study; therefore, the subsequent experiments can more accurately reflect the regulatory role of miR-221. Real-time RT-PCR analysis of the remaining samples demonstrated that miR-221 expression is up-regulated in 90\% of CRC samples, and it may be expected that miR-221 targets belong to the class of tumor suppressor genes or genes encoding proteins with potential tumor suppressor functions. Moreover, miR-221 expression is related to not only TNM stage but also tumor invasiveness. The level of miR-221 expression at TNM stages III and IV is higher than that at stages I and II, and the tumors invading adjacent tissues or organs display higher miR-221 levels than those limited to the wall of the colon and the rectum. Thus, miR-221 is likely to be an important oncomiR that can cleave or inhibit the target tumor suppressor mRNAs and that plays a role in CRC progression. In our study, CDKN1C/p57 protein expression is down-regulated in CRC, which was confirmed by Western blot analysis. Thus, it could be inferred that miR-221 binds to the 3'-UTR of the CDKN1C/p57 mRNA in an incomplete complementary pairing pattern. Therefore, it could only inhibit the further translation of the CDKN1C/p57 mRNA without inducing complete degradation, consistent with the result that the CDKN1C/ p57 mRNA levels in CRC and non-tumorous tissues are not significantly different, whereas CDKN1C/p57 protein expression decreases in CRC. It could also be inferred that tumor cells probably gain a proliferative advantage through miR221-mediated post-transcriptional silencing of CDKN1C/p57. Therefore, our data indicate the presence of more than one mechanism regulating CDKN1C/p57 expression in CRC.

Our results also demonstrated that miR-221 is dramatically up-regulated in four human CRC-derived cell lines compared with that in HUVEC, and this is in accordance with the results from clinical samples. Indeed, $\mathrm{CDKN} 1 \mathrm{C} / \mathrm{p} 57$ is down-regulated in response to miR-221 transfection in CRC cells, and a significant up-regulation of CDKN1C/p57 occurs in response to anti-miR-221 transfection. A direct interaction between miR-221 and the target site in the $3^{\prime}$-UTR of the CDKN1C/p57 mRNA was also demonstrated by the luciferase assay. By controlling CDKN1C/p57 expression, miR-221 upregulation promotes the growth of CRC cells by increasing the S-phase population. Conversely, miR-221 inhibition represses CRC cell growth and induces cell apoptosis. Cell cycle progression and cell apoptosis are usually closely associated. Cells failing to progress to the mitosis phase are destined for apoptosis. Aside from cell cycle arrest, the inhibition of cell 
growth by anti-miR-221 observed in CRC cells may also result from increased apoptosis. In this study, at $48 \mathrm{~h}$ anti-miR-221 transfection, cells were arrested at the $G_{0} / G_{1}$ phase and blocked from entering the $S$ phase. As seen in other tumor cells, we demonstrate that anti-miR-221 induces significant apoptosis in CRC cells.

Recently, it has been reported that there are CDKN1C/ p57-independent effects of miR-221 in tumorigenesis ${ }^{[22]}$. Moreover, miR-221 possesses many oncogenic functions other than serving as a CDKN1C/p57 inhibitor ${ }^{[23]}$. In this study, we showed that a miR-221-specific inhibitor, anti-miR-221, can inhibit CRC cell proliferation. Using a CDKN1C/p57-specific antagonist, anti-p57-siRNA, we demonstrated that the inhibitory effect of anti-miR-221 on CRC cell growth is largely, but not completely, mediated by CDKN1C/p57, suggesting that miR-221 might regulate $\mathrm{CDKN1C/p57-independent} \mathrm{signaling} \mathrm{pathways}$ to promote $C R C$ growth. It has been previously shown that CDKN1B/p27 is also a miR-221 target and that CDKN1B/ p27 expression consistently inversely correlates with miR221 overexpression in most cancers, which may explain why the inhibition of anti-miR-221 on CRC cell growth is only partly abrogated by anti-p57-siRNA ${ }^{[24]}$. Interestingly, it has also been reported that c-Kit, a common oncogene, is a miR-221 target in thyroid papillary carcinoma ${ }^{[25]}$. However, the role of c-Kit expression in CRC cell proliferation and carcinogenesis remains undefined. Our results, which identified CDKN1C/p57 as a miR-221 target in CRC cell lines, perfectly fit the dynamic view of the miRNA-mediated regulation of gene expression: the relationship between miRNAs and target mRNAs is not a "one to one" connection because the same mRNA can be regulated by more than one miRNA, and the choice of how many and which miRNAs target an individual $3^{\prime}$-UTR is largely determined by the specific cellular environment ${ }^{[26]}$. A miRNA that regulates target genes playing opposite roles in regulating cell proliferation may act as a tumor suppressor in some cancers but as an oncogene in others, depending on which targets are driving tumorigenesis in that specific cellular milieu.

In summary, we identified miR-221 as a direct regulator of CDKN1C/p57 expression in CRC, demonstrating a new mechanism for CDKN1C/p57 down-regulation in CRC. These findings further emphasize the importance of miR-221 in CRC carcinogenesis. However, it is noteworthy that the results in this study are based on cultured CRC cells that might not necessarily comprehensively reflect the in vivo situations. Therefore, further experiments are required to elucidate the antitumor mechanisms of anti-miR-221 in in vivo systems.

\section{Acknowledgements}

This work was supported by the Natural Science Foundation of Guangdong Province (№ 8451051501000390), the Medical Scientific Research Foundation of Guangdong Province (№ B2010190) and the Chief Foundation of Nanfang Hospital of Southern Medical University (№ 2008C005).

\section{Author contribution}

Dr Kai SUN designed the research, analyzed the data, wrote the manuscript and revised the paper; Wei WANG, Jun-jie ZENG, Cheng-tang WU, and Shang-tong LEI performed the experiments; Guo-xin LI assisted with the experiments.

\section{References}

1 Motoyama K, Inoue H, Takatsuno Y, Tanaka F, Mimori K, Uetake H, et al. Over- and under-expressed microRNAs in human colorectal cancer. Int J Oncol 2009; 34: 1069-75.

2 Wang CJ, Zhou ZG, Wang L, Yang L, Zhou B, Gu J, et al. Clinicopathological significance of microRNA-31, -143 and -145 expression in colorectal cancer. Dis Markers 2009; 26: 27-34.

3 Yantiss RK, Goodarzi M, Zhou XK, Rennert H, Pirog EC, Banner BF, et al. Clinical, pathologic, and molecular features of early-onset colorectal carcinoma. Am J Surg Pathol 2009; 33: 572-82.

4 Guarnieri DJ, DiLeone RJ. MicroRNAs: a new class of gene regulators. Ann Med 2008; 40: 197-208.

5 Cheng HY, Obrietan K. Revealing a role of microRNAs in the regulation of the biological clock. Cell Cycle 2007; 6: 3034-5.

6 La Rocca G, Badin M, Shi B, Xu SQ, Deangelis T, Sepp-Lorenzinoi L, et al. Mechanism of growth inhibition by MicroRNA 145: the role of the IGF-I receptor signaling pathway. J Cell Physiol 2009; 220: 485-91.

7 Shin S, Cha HJ, Lee EM, Jung JH, Lee SJ, Park IC, et al. MicroRNAs are significantly influenced by $\mathrm{p} 53$ and radiation in HCT116 human colon carcinoma cells. Int J Oncol 2009; 34: 1645-52.

8 Yamamichi N, Shimomura R, Inada K, Sakurai K, Haraguchi T, Ozaki $\mathrm{Y}$, et al. Locked nucleic acid in situ hybridization analysis of miR-21 expression during colorectal cancer development. Clin Cancer Res 2009; 15: 4009-16.

9 Fornari F, Gramantieri L, Ferracin M, Veronese A, Sabbioni S, Calin GA, et al. MiR-221 controls CDKN1C/p57 and CDKN1B/p27 expression in human hepatocellular carcinoma. Oncogene 2008; 27: 5651-61.

10 Diaz-Meyer N, Day CD, Khatod K, Maher ER, Cooper W, Reik W, et al. Silencing of CDKN1C(p57/KIP2) is associated with hypomethylation at KvDMR1 in Beckwith-Widemann syndrome. J Med Genet 2003; 40: 797-801.

11 Vlachos P, Joseph B. The Cdk inhibitor p57(Kip2) controls LIM-kinase 1 activity and regulates actin cytoskeleton dynamics. Oncogene 2009; 28: 4175-88.

12 Bartels CL, Tsongalis GJ. MicroRNAs: novel biomarkers for human cancer. Clin Chem 2009; 55: 623-31.

13 Sun K, Wang Q, Huang XH. PPAR gamma inhibits growth of rat hepatic stellate cells and TGF beta-induced connective tissue growth factor expression. Acta Pharmacol Sin 2006; 27: 715-23.

14 Mirnezami AH, Pickard K, Zhang L, Primrose JN, Packham G. MicroRNAs: key players in carcinogenesis and novel therapeutic targets. Eur J Surg Oncol 2009; 35: 339-47.

15 Baffa R, Fassan M, Volinia S, O'Hara B, Liu CG, Palazzo JP, et al. MicroRNA expression profiling of human metastatic cancers identifies cancer gene targets. J Pathol 2009; 219: 214-21.

16 Schetter AJ, Harris CC. Plasma microRNAs: a potential biomarker for colorectal cancer? Gut 2009; 58: 1318-9.

17 Ahmed FE, Jeffries CD, Vos PW, Flake G, Nuovo GJ, Sinar DR, et al. Diagnostic microRNA markers for screening sporadic human colon cancer and active ulcerative colitis in stool and tissue. Cancer Genomics Proteomics 2009; 6: 281-95.

18 Glinskii AB, Ma J, Ma S, Grant D, Lim CU, Sell S, et al. Identification of intergenic trans-regulatory RNAs containing a disease-linked 
SNP sequence and targeting cell cycle progression/differentiation pathways in multiple common human disorders. Cell Cycle 2009; 8: 3925-42.

19 Matsuoka S, Edwards MC, Bai C, Parker S, Zhang P, Baldini A, et al. p57KIP2, a structurally distinct member of the p21CIP1 Cdk inhibitor family, is a candidate tumor suppressor gene. Genes Dev 1995; 9: 650-62.

20 Soejima H, Nakagawachi T, Zhao W, Higashimoto K, Urano T, Matsukura $\mathrm{S}$, et al. Silencing of imprinted CDKN1C gene expression is associated with loss of $\mathrm{CpG}$ and histone $\mathrm{H} 3$ lysine 9 methylation at DMR-LIT1 in esophageal cancer. Oncogene 2004; 23: 4380-8.

21 Galardi S, Mercatelli N, Giorda E, Massalini S, Frajese GV, Ciafrè SA, et al. MiR-221 and miR-222 expression affects the proliferation potential of human prostate carcinoma cell lines by targeting p27Kip1. J Biol Chem 2007; 282: 23716-24.

22 Felli N, Fontana L, Pelosi E, Botta R, Bonci D, Facchiano F, et al.
MicroRNAs 221 and 222 inhibit normal erythropoiesis and erythroleukemic cell growth via kit receptor down-modulation. Proc Natl Acad Sci USA 2005; 102: 18081-6.

23 Le Sage C, Nagel R, Egan DA, Schrier M, Mesman E, Mangiola A, et al. Regulation of the $\mathrm{p} 27$ (Kip1) tumor suppressor by miR-221 and miR222 promotes cancer cell proliferation. EMBO J 2007; 26: 3699708.

24 Visone R, Russo L, Pallante P, De Martino I, Ferraro A, Leone V, et al. MicroRNAs (miR)-221 and miR-222, both overexpressed in human thyroid papillary carcinomas, regulate p27Kip1 protein levels and cell cycle. Endocr Relat Cancer 2007; 14: 791-8.

$25 \mathrm{He} \mathrm{H}$, Jazdzewski K, Li W, Liyanarachchi S, Nagy R, Volinia S, et al. The role of microRNA genes in papillary thyroid carcinoma. Proc Natl Acad Sci USA 2005; 102: 19075-80.

26 Meltzer PS. Cancer genomics: small RNAs with big impacts. Nature 2005; 435: 745-6. 